\title{
LA VIRTUALIZACIÓN EN LA PERSPECTIVA DE LA MODERNIZACIÓN DE LA EDUCACIÓN SUPERIOR: CONSIDERACIONES PEDAGÓGICAS
}

Angel H. Facundo Díaz.

INTRODUCCIÓN

La preocupación por educar, empleando los medios y mediaciones más eficientes y adecuados posibles, es tan vieja como el ser humano. Más aún: la historia universal de la educación muestra claramente que, a medida que se han desarrollado nuevas tecnologías de información y comunicación, se han realizado los más significativos avances en materia de educación y, sobre todo, de pedagogía ${ }^{1}$.

En buena medida, los esfuerzos periódi-

\section{RESUMEN}

Desde diversos ejes temáticos: interrelaciones tecnología-pedagogía; modelos pedagógicos e institucionales; paradigmas y rupturas; y la calidad de la educación, el autor hace un detallado diagnóstico acerca del sistema educativo colombiano y devela las fallas del mismo enfrentado a los procesos de autoevaluación y acreditación de la educación superior.

Como resultado, resalta la importancia de la educación virtual y a distancia como una herramienta pedagógica en la formación de investigadores con un aprendizaje autónomo. Además, ve la necesidad de elaborar pedagogías que preparen a los estudiantes para la inserción global en la actual sociedad del conocimiento.

Este texto permite entender los retos a los que se enfrenta el sistema educativo colombiano, además de sus posibilidades en la búsqueda del mejoramiento de la calidad en la educación superior.

\section{ABSTRACT}

From diverse core themes: technology- teaching interrelations; pedagogic and institutional models; paradigms and ruptures; and the quality of education, the author makes a thorough diagnosis of the Colombian education system uncovering its errors as compared to the selfassessment and accreditation processes of higher education.

As a result thereof, it highlights the importance of virtual learning and distance education as a pedagogical tool in training researchers through an autonomous learning. Furthermore, he sees the need to create teaching aimed at preparing students for global integration to present knowledge society. This is an essential text to understand the targets faced by the Colombian education system, as well as the existing possibilities in the search for quality improvement within higher education.

1 Si bien el término "pedagogía" etimológicamente se refiere específicamente a la educación de los menores, y existen diferencias en materia de educación de adultos que se expresan bajo el término "andragogía", aquí se empleará el primer término en sentido amplio, comprendiendo genéricamente uno y otro conceptos específicos (pedagogía/andragogía). Se advertirá expresamente, cuando se emplee dicho término en forma específica. 
cos por "modernizar", no son otra cosa que poner a tono la educación con las exigencias y desarrollos tecnológicos, conceptuales y pedagógicos de cada época, de tal forma que pueda satisfacer con calidad y de forma más equitativa y pertinente las necesidades de desarrollo humano y social de cada cultura, civilización y sociedad ${ }^{2}$.

Sin embargo, como en la mayoría de los diferentes campos de la actividad humana, estos avances no han sido generalizados ni han avanzado siempre parejos. Por el contrario. Si se analizan detenidamente las interrelaciones entre tecnologías y pedagogía, a lo largo de la historia de la civilización humana se hacen evidentes marcados desfases de la pedagogía en relación con los avances tecnológicos, siendo casi siempre aún mayor la brecha en los denominados países en desarrollo.

Una de las formas más prácticas que he encontrado a lo largo de mis años dedicados al tema de la calidad de la educación ha sido precisamente señalar las interrelaciones y desfases entre tecnología y pedagogía, ya que ellas permiten encontrar de forma más precisa y balanceada las diferentes acciones por desarrollar, facilitan la focalización de esfuerzos frente a los siempre escasos recursos disponibles y, sobre todo, evitan caer en la tentación, tantas veces irresistible, de considerar que "modernización" sea sólo sinónimo de actualización tecnológica, sin acometer los previos y necesarios avances en la elaboración de nuevos enfoques teóricos, pedagógicos e institucionales, sin los cuales aquellos no pueden producir los resultados esperados. En los últimos años, me han oído repetir en los más diferentes escenarios la necesidad de enfrentarnos a lo que denomino la necesidad de elaborar una "pedagogía basada en las tecnologías virtuales" y de hacer "reingeniería institucional". Por supuesto, también aquí pretendo hacerlo, y a dichas interrelaciones dedicaré las primeras reflexiones.

De otra parte, si bien el foco específico de atención de esta conferencia es la educación a distancia, quiero dedicarme en un segundo momento a presentar algunos de los cambios o rupturas que visualizo en los tradicionales modelos pedagógicos e institucionales y que considero son válidos para cualquier modalidad educativa: tanto presencial como a distancia. De hecho, ésta como toda separación es más ficticia que real, y en la actualidad con la educación virtual entramos por fortuna en proceso de convergencias.

En un tercer punto, haré unas breves consideraciones sobre el problema de la calidad de la educación en la actual sociedad del conocimiento de este pequeño mundo globalizado actual. Si no concentramos nuestros mejores esfuerzos en garantizar calidad, seremos cada vez más desplazados por aquellas instituciones educativas que, sin importar su origen, sí ofrezcan importantes niveles de calidad. Ese es -sin duda- el mayor reto contemporáneo de nuestras instituciones: un reto de verdadera supervivencia.

Finalmente, realizaré unos pocos comentarios sobre la evaluación y acreditación de la educación ya que éstos son dos de los principales mecanismos para garantizar la calidad.

Aunque ha sido menospreciada y quizás no haya podido avanzar tanto como se

2 Cfr. Facundo, A. y C. Rojas (1990). La calidad de la educación: cómo entenderla y evaluarla. Bogotá: FESAlegría de Enseñar. 
hubiera querido, la llamada educación a distancia presenta en la actualidad las mayores posibilidades para liderar la “modernización” de la educación superior, o mejor aún, la necesaria transformación y cambio paradigmático que nos facilitan las tecnologías digitales y nos exige la sociedad del conocimiento. Desde hace ya varias décadas en la educación a distancia se ha venido hablando e implementando con mayor o menor éxito la apertura y flexibilidad de los currículos.

Si se logran desmontar en una y otra modalidad esas "pautas habituales de conducta", esos "errores pedagógicos" (como los denomina Otto Peters), -que consideran que el aprendizaje es producto de la enseñanza y que ésta consistiría en la entrega secuencial y dirigida de contenidos que, dicho sea de paso, estamos extendiendo a la era digital y lo estamos haciendo con "especial énfasis y habilidad"3 tecnológicas-, la educación virtual, es decir la educación que hace uso de todas las posibilidades técnicas actualmente existentes, podrá contribuir a realizar la también vieja aspiración de tantos educadores de enseñar a aprender de forma autónoma y flexible, a lo largo de toda la vida. Esa para mí sería la verdadera “modernización” de la educación.

\section{LAS INTERRELACIONES TECNOLOGÍA - PEDAGOGÍA.}

Cuando se analiza con detenimiento la historia de la educación, se encuentra que los grandes avances en pedagogía han sido producidos fundamentalmente gracias a cambios tecnológicos, que, a su vez, no son otra cosa que la aplicación de resultados de cambios en la manera de entender y ejercer dominio sobre la realidad. Estos cambios conllevan -siempre que se los aplica adecuadamente- verdaderas transformaciones en la totalidad de los paradigmas existentes. La educación que es una de las más típicas actividades del conocimiento, no ha estado ni puede estar ajena a dichos cambios.

Hagamos un muy rápido recorrido histórico para tratar de ilustrar estas interrelaciones. La educación es tan antigua como el ser humano, y ha evolucionado con él a lo largo de la historia. La sociología de la educación la define como una compleja relación de comunicación, por medio de la cual el ser humano "se enfrenta interactiva-
Cuando se analiza con detenimiento la historia de la educación, se encuentra que los grandes avances en pedagogía han sido producidos fundamentalmente gracias a cambios tecnológicos, que, a su vez, no son otra cosa que la aplicación de resultados de cambios en la manera de entendery ejercer dominio sobre la realidad.

\section{RESEÑA DE AUTOR:}

Licenciado en Filosofía de la Universidad Nacional de Colombia; Sociólogo Diplomado (Diplom Soziologe) y Doctor Philosophiae (Ph.D.) de la Universidad Humboldt de Berlín, Alemania. Se ha desempeñado como investigador/consultor, catedrático universitario y conferencista invitado en diversas instituciones y universidades. Su línea principal de investigación es sobre la calidad de la educación y factores asociados. De ella ha derivado dos líneas secundarias: tecnologías digitales de información y comu- nicación aplicadas a la educación (educación virtual superior); e incidencia de la alimentación escolar en el rendimiento académico y otros campos.

Autor de diversos trabajos y publicaciones en el campo de la educación superior virtual, entre ellos: "La educación a distancia/virtual en Colombia" (2004), "La educación superior abierta y a distancia: necesidades para su establecimiento y desarrollo en América Latina y el Caribe" (2004) y "Crecimiento y desarrollo educativo en Colombia, 1958-1993" (1994).

3 Peters, Otto (2002). La educación a distancia en transición. Nuevas tendencias y retos. México: Innova, Universidad de Guadalajara. 
mente" a la realidad -tanto exterior como interior- $y$, mediante el proceso de conocimiento, "aprehende", es decir, "extracta y se apropia” de sus características esenciales, las "interioriza como conocimientos", las “enseña" y comparte con otros, es decir, las "pone a disposición" de los demás con quienes interactúa, sintetiza, analiza, aplica y perfecciona; y en ese proceso crece en sus estructuras y niveles de conciencia, "se autoconstruye" permanentemente, ampliando así las potencialidades tanto individuales como sociales.

El desarrollo personal, cultural y social es el resultado, o sea, la cristalización de los diversos procesos de conocimiento. Si bien, el diagrama anexo está excesivamente simplificado, en él puede advertirse claramente cómo a medida que el ser humano ha desarrollado y ha podido disponer de nuevas tecnologías de información y comunicación, ha avanzado en sus formas de aprender, enseñar y construir conocimientos.

\section{Gráfica No.1}

Interelaciones Tecnología - Pedagogía

\begin{tabular}{|c|c|c|}
\hline 2002 & Percepción digital & Holografía multidimensional \\
\hline 1990 & WEB & Cognición \\
\hline 1983 & Internet & Interactividad/glocalidad \\
\hline 1977 & PCs & Tecnologías Digitales \\
\hline 1957 & Videograbadora & $\begin{array}{l}\text { Educ. a Distancia (Radio y TV) } \\
\text { Conductismo, Constructivismo } \\
\text { Educ. a Distancia (Impresos) } \\
\text { Pedagog. Activa }\end{array}$ \\
\hline 1920 & Electrónica, TV & Escuelas y U. públicas \\
\hline 1888 & Radio & Seminario \\
\hline 1882 & Electricidad & Libro Impreso \\
\hline 1450 & Imprenta & Escuelas o U. conventuales \\
\hline Siglo XII & Papel en Europa & $\begin{array}{l}\text { Liceos } \\
\text { Enseñanza frontal } \\
\text { "Objetivación" del conocimiento }\end{array}$ \\
\hline 50 a.C. & $\begin{array}{l}\text { Códices y papel } \\
\text { en China }\end{array}$ & \\
\hline 1800 a.C. & Alfabeto & \\
\hline
\end{tabular}

En el diagrama puede apreciarse claramente el largo camino del conocimiento. Si el lenguaje oral permitió la aparición de la enseñanza, la escritura es la tecnología que permite, entre otros muchos desarrollos, objetivar el conocimiento; almacenarlo en forma material, independiente del sujeto, el surgimiento de nuevas profesiones especializadas en "transmitirlo" a ámbitos mayores: el escribano y el maestro; la creación de un método menos individual y masivo de enseñanza; y la aparición de una institución especializada en socializar los conocimientos disponibles: la escuela. La imprenta, permite una mayor flexibilidad en el almacenamiento y distribución material objetivada del conocimiento, posibilita igualmente su profundización y expansión. Por eso Humboldt, al advertir estas posibilidades (hoy diríamos "virtualidades") ofrecidas por la imprenta, crea el seminario, como método para ir más allá de lo dicho en las enseñanzas del maestro por medio del método de la exposición que no era otra cosa que leer los grandes libros manuscritos frente a los alumnos (en alemán Vorlessung), ir a las diversas fuentes, superando el "timeo hominem unius libri”, profundizar mediante el debate libre, y así avanzar tanto en la fundamentación como en el desarrollo de los conocimientos disponibles. En una palabra, superar la simple función reproductora de la escuela y pasar a una función de producción de nuevo conocimiento, es decir posibilitar la investigación.

Sin embargo, este cambio no era fácil. Exigía disponer de la nueva tecnología: los libros impresos y las bibliotecas, y de una mayor exigencia para los docentes, quienes debían conocer la bibliografía existente y 
dedicar muchas horas a la lectura de los más extensos volúmenes de libros impresos. Por eso quizás no pudo extenderse este nuevo método pedagógico, sobre todo en países como los nuestros y, luego de la aparición y el desarrollo del libro, nos quedamos "presos" en el viejo modelo de escuela, de docentes y de pedagogía. Surgen entonces los desfases entre tecnología y pedagogía.

Estos se hacen más crecientes con la aparición de los nuevos medios audiovisuales. Para la mayoría de nuestras instituciones educativas era económicamente difícil y poco prioritario invertir en su adquisición. La brecha entre tecnologías y pedagogías se hacen crecientes hasta llegar a las nuevas tecnologías de información y comunicación (NTCI). Así las cosas, desaprovechamos ese creciente proceso de objetivación, flexibilización y por consiguiente crecimiento del conocimiento que gradualmente van permitiendo las sucesivas tecnologías. Y nuestra educación queda -en buena medidadesfasada no sólo en términos de metodologías pedagógicas, sino de acceso a las crecientes ventajas del conocimiento, hasta llegar a una sociedad como la actual, en la cual se convierte en el factor más dinámico de desarrollo individual y social.

Por supuesto que los cambios no han sido nunca fáciles. Las tecnologías son artefactos, bien sea físicos o semióticos, que implican nuevos lenguajes y dominios que exigen nuevos esfuerzos que significan incomodidades y costos, y en no pocas ocasiones irritaciones y resistencias. En la mayoría de los casos éstas hacen que se rechacen. En otros, se aceptan e interiorizan parcialmente, se producen acomodos, mezclas y coexistencias con desarrollos anteriores. En un número menor, se efec- túan las necesarias rupturas, reorientaciones y reestructuraciones, se crean y socializan los nuevos modelos, es decir, se cambia formas de pensar y actuar.

La historia del subdesarrollo no es otra cosa que la historia de los desfases entre tecnologías y educación. Sin embargo, como nunca antes, en la actualidad contamos de nuevo con la posibilidad de ponernos a tono con la historia, si sabemos acceder a la denominada educación virtual, aprovechando de manera integral toda la amplia gama de posibilidades que nos ofrecen las tecnologías digitales (eso es lo que en esencia significa el término "virtual") y, a semejanza de lo que hizo Humboldt en su época, desarrollamos nuevas pedagogías, mediaciones e instituciones que le sean acordes.

\section{MODELOS PEDAGÓGICOS E INSTITUCIONALES: PARADÍGMASY RUPTURAS}

Sobre la necesidad de las rupturas y cambios, que no son otra cosa que la adopción de nuevos paradigmas y modelos, se ha venido insistiendo recurrentemente. Estos son múltiples. Sin embargo, yo relevo dos de ellos: cambios en los modelos pedagógicos y una reingeniería de las instituciones educativas, para hacerlas con las actuales tecnologías digitales. Uno y otro se incluyeron en la llamada Declaración de Quito.

Estas rupturas no han sido ni son fáciles. Ilustraré este punto con un ejemplo utilizado por Peters. Cuando la tecnología de la escritura comenzaba a cambiar el paradigma de la enseñanza y los aprendizajes verbales, "el propio Platón criticaba este cambio, alegando que la palabra escrita no podía responderle al estudiante que lee".

Sé que existen honrosas excepciones institucionales y un amplio número de docen-
La historia del subdesarrollo no es otra cosa que la historia de los desfases entre tecnologías y educación. Sin embargo, como nunca antes, en la actualidad contamos de nuevo con la posibilidad de ponernos a tono con la historia, si sabemos acceder a la denominada educación virtual. 
tes que han batallado desde siempre por producir cambios reales en la educación. Sin embargo, en el larguísimo letargo, en el cual han vivido nuestras escuelas y universidades, aún no hemos podido superar el paradigma de las clases, las conferencias, la interacción verbal, como si no hubieran aparecido sucesivamente las diversas tecnologías y artefactos como la imprenta y el modelo pedagógico del "seminario alemán” creado por Humboldt, que basa el aprendizaje precisamente en la consulta e indagación bibliográficas.

Más aún: todavía la atención y seguridad de no pocos maestros y estudiantes se sigue fundamentando en la "enseñanza por exposición y el aprendizaje receptivo", siguiendo un currículo único, previamente definido, en el cual, mediante la transmisión de contenidos, repartidos y entregados gradualmente a lo largo de un curso y la realización de determinadas tareas, éstos han de ser "aprehendidos" -muchas veces tan sólo memorizados-, como forma de garantizar que se alcanzaron unos determinados "objetivos de aprendizaje", previstos en un "diseño curricular", no pocas veces definido "a priori”, sin mayor análisis ni confrontación.

Sin embargo, ese modelo tradicional aceptado por muchos como "natural", puesto que aparentemente no usaría artefactos o puesto que se consideraba el proceso típico en los aprendizajes, no lo es en realidad.

De una parte, ya hace bastante tiempo se conoce que también el lenguaje oral es un artefacto semiótico, un verdadero medio y una mediación creados y utilizados por el ser humano. Se conoce que toda educación es mediada. Quizás con el computador, que antes que medio de enseñanza-aprendizaje lo es de producción, comunicación, indagación, investigación, diversión, juego; en fin, que se utiliza en casi todas las esferas de la vida, estamos dando - por fin- a los medios y a las mediaciones la importancia que antes desconocíamos o negábamos. Y, con ellos, la pedagogía y la didáctica han recobrado un nuevo valor. En ellas se están finalmente destacando diversas características antes subvaloradas: la indagación, la investigación, la experiencia interactiva en sus diversas formas y medios, el análisis contextual, la flexibilidad dentro de reglas, diferentes estrategias y métodos, como la simulación y lo lúdico ${ }^{4}$, antes sólo aceptados en el caso de la educación infantil.

De otra parte, cuando se analizan detallada y sistemáticamente los procesos de aprendizaje, se encuentra que la mayoría de ellos no se producen de forma lineal y secuencial y gracias a las "exposiciones" y a la "ayuda" de un maestro, sino por diversas rutas de exploración y búsqueda, casi siempre relativamente autónomas y nosecuenciales, que siguen diversos caminos, impredecibles, abiertos, flexibles, pluralistas, eclécticos y, en no pocas ocasiones, de forma "casual" y no causal. Ese sí es el camino natural del aprendizaje: aprender por indagación o investigación.

Estas no son características típicas sólo de la educación que se apoya en computadores, sino de toda educación. Las tecnologías digitales han facilitado su compren-

4 Galvis, Alvaro . "CLIC en la didáctica: oportunidad de enseñar y aprender mediante experiencias, indagación, reflexión y socialización con apoyo en tecnología”. En: Revista Tecnología y Comunicación Educativas, No. 37, enero-junio (2003). 
sión, pero sobre todo su dominio, gracias a la disponibilidad e integración de los diversos medios: el hipertexto, la multimedia, las veloces herramientas de búsqueda, almacenamiento, recuperación, manipulación y análisis de información, de comunicación interactiva y en redes, la simulación, la realidad virtual y tantas otras que nos brindan en la actualidad los computadores y las denominadas NTCI.

Con todo, no se trata de avances meramente instrumentales, de disponer y utilizar los nuevos artefactos o medios tecnológicos, sino de cambios conceptuales y culturales más profundos. Gracias al uso de las tecnologías se han venido tomando conciencia de ciertos elementos antes ignorados (por ejemplo, del espacio de aprendizaje y de la necesaria multidimensionalidad de la programación curricular ya que existen diversos tipos de inteligencia, de habilidades y personalidad en quien aprende. Esta toma de conciencia ha sido facilitada por los medios tecnológicos y por las investigaciones y nuevos conocimientos sobre el funcionamiento reticular del cerebro, sobre la comunicación y el proceso de aprendizaje, y ha permitido (o está permitiendo) acelerados y abruptos cambios en los enfoques y diseños curriculares, y en las prácticas educativas.

Por supuesto que el paso de un enfoque tradicional lineal, preconfigurado, rígido y ligado a espacios "ritualistas" de aprendizaje, en los cuales se ha desarrollado una estructura educativa que se basa en enseñanza por exposición, en aprendizaje receptivo y en una interacción espacio, tiempo y sujeto, hacia enfoques más flexibles, abiertos, dinámicos, impredecibles, pluralistas, dinámicos, interactivos, transdisciplinarios, donde el tiempo y el espacio desaparecen, como lo preveía la física cuántica, y toman lugar predominante en la conciencia de una manera expandida y dinámica, no ha sido ni es fácil aunque hubieran evidencias de su beneficio. Por ejemplo, en las "cogniciones esotéricas" tan típicas en el mundo oriental; en las hipótesis holográficas multidimensionales de la física cuántica; en el desarrollo "pensamiento lateral" frente al pensamiento causal; en los acelerados progresos de la educación a distancia; o incluso en los más recientes resultados de la neurofisiología contemporánea sobre la reticularidad y tiempos diferenciales de respuestas de las diferentes capas del cerebro o sobre las inteligencias múltiples.

En la pasada conferencia internacional de Innova, que año a año se realiza durante la Feria Internacional del Libro en Guadalajara, México, yo utilizaba la figura de los modelos tradicionales de educación -bien sea de la educación convencional presencial o de las primeras etapas de la educación a distancia- que han obligado al ser humano a introducirse como en un "túnel educativo", donde su gran riqueza multidimensional ha sido dejada de lado al privilegiar, casi exclusivamente, el modelo de la mediación oral tradicional de las pedagogías de exposición-recepción ${ }^{5}$. Sin embargo, luego de un largo y oscuro camino, las diversas tecnologías análogas (imprenta, radio, cine, televisión), permitieron gradualmente ampliar ese túnel,
Con las nuevas tecnologías los currículos han de ser propuestas flexibles, de exploración por diversos caminos, muchas veces impredecibles que, como lo expresaba Machado, se construyen al andar, en el propio proceso de búsqueday aprendizaje.

5 Para designar el modelo pedagógico tradicional utilizo la terminología empleada por David Ausbel en sus dos magistrales libros. Cfr. Ausbel, D.P. The Psycology of Meaningful Verbal Learning. Nueva York: Gruñe y Straton, 1968. Y Ausbel. D.P. Pyicologie des Unterrichts. Weinheim: Belz, 1980. 
El aprendizaje

sedaen

espacios

virtuales que,

en criterio

de diversos

autores ha sido

una ruptura

clave, un

"acontecimien-

to revoluciona-

rio", un "punto

decisivo de

magnitud

copernicana",

el "evento

pedagógico más

fundamental

de la

actualidad". hasta que con las nuevas tecnologías digitales, que son la integración del computador, la multimedia y las redes, se inició finalmente su ruptura definitiva y, con ello, la liberación del ser humano de esa camisa de fuerza educativa que imponían los métodos expositivo-receptivo orales, ligados a espacios de aprendizajes reales pero rígidos, para permitir el despliegue a plenitud de las capacidades, aprendiendo de forma autónoma y autorregulada en espacios virtuales flexibles y dinámicos.

Para expresarlo en pocas palabras y en términos pedagógicos, con la aparición de las tecnologías digitales que permiten el almacenamiento, la exploración y recuperación "autónomas" de información, así como una presentación multiforme e hipertextual, la simulación y manipulación multidimensional de la realidad y las más diversas formas de intercomunicación y colaboración, ya los currículos no pueden continuar siendo los cursos lineales, dirigidos por otros, con contenidos prefabricados y únicos para un gran número de estudiantes que, insisto, es la forma fundamental en que hemos venido realizando los procesos educativos tanto presenciales como en la educación a distancia, durante sus primeras etapas de desarrollo.

Con las nuevas tecnologías los currículos han de ser propuestas flexibles, de exploración por diversos caminos, muchas veces impredecibles que, como lo expresaba Machado, se construyen al andar, en el propio proceso de búsqueda y aprendizaje. El aprendizaje ya no puede seguir siendo una "consecuencia" de la enseñanza, sino un proceso fundamentalmente autónomo, de autodescubrimiento, realización y transformación. El estudiante deja de ser considerado como un "objeto", un recipiente o un usuario de instrucción y pasa a ser el sujeto responsable de su propio trabajo de aprendizaje y autodesarrollo. El aprendizaje se da en espacios virtuales que, en criterio de diversos autores ha sido una ruptura clave, un "acontecimiento revolucionario", un "punto decisivo de magnitud copernicana", el "evento pedagógico más fundamental de la actualidad"8.

No cabe duda de que, si bien la educación presencial convencional todavía no es, ni mucho menos, "una categoría residual", como lo pronostica Rolf Schulmeister ${ }^{9}$ (en buena medida a causa de la resistencia de maestros y estudiantes), sí son crecientes el entusiasmo y la creación de programas de educación virtuales para estudiantes alejados de los grandes centros, así como la incorporación gradual de procesos de aprendizaje en línea en programas de educación presencial. Incluso aquellas instituciones que por diversas razones se opusieron a la educación a distancia clásica, hoy han adoptado las tecnologías digitales y utilizan estos ambientes para la enseñanza-aprendizaje. Y esto no sólo sucede en otras latitudes. Se ha venido dando de una manera acelerada en nuestra región. Las investigaciones nacionales y el estudio regional sobre universidad virtual que se han venido realizando por encargo de la Unesco-Iesalc lo han podido constatar.

6 Perelman, L.J. School's out. A Radical New Formula for the Revitalization of America's Educational System. New York: Avon Books, 1992.

7 Kleinschrith, R. Neues Lernen mit dem Computer. Reinbeck: Rowohlt, 1996.

8 Peters, O. Op. Cit., pág. 135.

9 Shulmeister, R. Virtuelle Universität. Virtuelles Lernen. Munich: Oldenbourg, 2001. 
Sin embargo, las tecnologías digitales por sí solas no explican las rupturas pedagógicas. Tan sólo las posibilitan. De hecho, durante los primeros años de su utilización -tanto por parte de la educación presencial como en las primeras etapas de la educación a distancia-, fue corriente continuar con los viejos modelos pedagógicos de exposición, aprendizaje programado y recepción pasiva. Y, aunque en menor escala, todavía continúan replicándose en la denominada educación virtual. Más aún, -siguiendo los modelos pedagógicos tradicionales- pueden encontrarse incluso sofisticados desarrollos técnicos que aprovechan las inmensas posibilidades que brindan las tecnologías digitales, para superar la monotonía de las presentaciones y lograr mejores enfrentamientos "icónicos", "simbólicos" e incluso más "activos" 10 de la realidad, mediante el uso de los multimedia. Peters califica estos desarrollos como "extender a la era digital las pautas habituales de conducta", y cometer "errores pedagógicos" con "especial énfasis y habilidad".

La ruptura de los viejos modelos pedagógicos requiere nuevos conceptos que se fundamentan en los grandes avances de las investigaciones sobre el cerebro y sobre las inteligencias múltiples (neurociencias y psicología cognoscitiva), la lingüística, las teorías de la comunicación y de los procesos de aprendizaje que, utilizando igualmente las grandes posibilidades de los ambientes digitales para enfrentar la realidad de forma “autónoma”, están transformando gra- dualmente -muchas veces de forma imperceptible- los viejos conceptos y modelos pedagógicos, los roles de los docentes de los agentes educativos (docentes, estudiantes, administradores), así como las estructuras mismas de las instituciones educativas y, en particular, de la universidad tradicional, tanto presencial como a distancia.

Así, dentro de este último modelo, cada vez se supera más la tradicional función del docente que prepara y "presenta contenidos" a sus estudiantes, clase tras clase, y se experimentan y desarrollan otros que, si bien son más complejos, exigentes y mediados, son más prometedores en materia de aprendizajes, en cuanto que éstos se dan en condiciones más naturales y reales del diario vivir, y en cuanto se obtienen autónomamente mediante indagación, investigación y descubrimiento, combinando técnicas de aprendizaje con técnicas de investigación, siguiendo el modelo de trabajo del investigador independiente ${ }^{11}$.

Estos nuevos métodos pedagógicos que pretenden romper con las viejas tradiciones, se fundamentan en el desarrollo de estudiantes autónomos. En la sociedad del conocimiento, como nunca antes, se requieren estudiantes activos que, por sí mismos, sean capaces de planificar, buscar, evaluar, aplicar y renovar constantemente los conocimientos a lo largo de toda la vida. Por tal razón, sobre unas mínimas propuestas o guías curriculares, se les brinda a los estudiantes total libertad para seleccionar los contenidos, las estrategias y medios por utilizar, las metas y obje-
En la sociedad del conocimiento, como nunca antes, se requieren estudiantes activos que, por símismos, sean capaces de planificar, buscar, evaluar, aplicar y renovar constantemente los conocimientos a lo largo de toda la vida.

10 De acuerdo con Jerome Bruner, existen tres métodos de confrontar y apropiarse de la realidad en los procesos de aprendizaje: mediante imágenes, esquemas, mapas, etc. (icónico); mediante conceptos, argumentos, pensamiento (simbólico); y el método activo. Cfr: Bruner, J. Entwurfeiner Unterricht Theorie: Berlín: Berlin Verlag, 1973.

11 Cfr. Peters, Op. Cit. Págs. 121-122. Yo mismo he venido experimentando estos modelos con mis alumnos de postgrado de la Universidad Javeriana. 
tivos específicos por perseguir, las formas de organizar, autoregular y analizar sus aprendizajes e, incluso, hasta de medir sus propios logros, de forma autónoma e independiente, todo ello, de acuerdo con sus intereses individuales. Estos nuevos modelos de trabajo académico (válidos tanto para estudiantes de educación presencial como a distancia) parten de unos pocos principios básicos, a saber: en la actualidad es el estudiante quien, de forma consciente, ha de llevar la responsabilidad por su aprendizaje; el conocimiento se encuentra disponible o hay que crearlo; se dispone de los medios técnicos de búsqueda e investigación que hacen cada vez más fácil el acceso a los conocimientos disponibles; $y$ se conocen los métodos fundamentales para evaluar los anteriores y crear nuevos conocimientos.

Por consiguiente, los nuevos métodos (y hablo siempre en plural porque existe una amplia gama de experiencias y propuestas), se centran cada vez más, aunque no exclusivamente, en el uso de las bibliotecas y bases de datos así como en el manejo creciente de medios digitalizados, particularmente de internet, para acceder a las fuentes de información. El principio y paradigma fundamental es aprender de forma consciente, por descubrimiento, indagando, compartiendo con otros sus problemas, análisis, conceptos e hipótesis "descubiertas” y haciendo investigación. En estos métodos, el docente pasa a cumplir las funciones de estimular y facilitar permanentemente a sus estudiantes en las labores de búsqueda y organización de los contenidos; de orientador y asesor de los aprendizajes autónomos.
No es fácil para los docentes, ni mucho menos para los estudiantes, desarrollar y acostumbrarse a los nuevos métodos, particularmente cuando hemos vivenciado e interiorizado otras formas de enseñanzaaprendizaje. Se requiere una alta dosis de motivación no siempre fácil de sostener, de conciencia, responsabilidad y actividad pero, sobre todo, un ejercicio permanente de reflexión crítica sobre el proceso mismo de aprendizaje (metacognición), así como de análisis, interpretación y evaluación de la información para poderla apropiar adecuadamente y transformarla en conocimientos. La larguísima tradición de no poder aprender, mejorar los conocimientos y desarrollar competencias "sin intervención externa" es un pesado lastre. Con todo, los logros y los niveles de eficacia que se alcanzan con los nuevos métodos hacen prever que más allá de una nueva "moda pedagógica", son la tendencia natural y sostenible de aprendizaje continuo a lo largo de la vida que, sin duda lugar a dudas, está contribuyendo a transformar todo el sistema educativo.

Por supuesto que, como lo indican autores de diversos países, estos métodos implican así mismo limitaciones. En ellos se trabaja relativamente en forma aislada ${ }^{12}$, se pierde la pertenencia y los efectos psicológicos que brindan los espacios, la convivencia y la interacción reales, en una palabra la dimensión histórica concreta que ofrecen los métodos tradicionales ${ }^{13}$. Por esa misma razón, quizás haya que diseñar sistemas educativos que combinen e integren -como proponen diversos autores- uno y

12 Turkle, S. Lebem im Netz. Identität im Zeiten des Internet. Reinbeck: Rowolt, 1998.

13 Cfr. Lewin, K. Feldtheorie des Lernens. En: Grauman, F.C (editor). Kurt Lewin Werkausgabe. Bern: Huber, 1998. 
otros modelos educativos: logrando más comunicación, interacción y colaboración presencial y menos exposición de contenidos ya que a éstos pueden alcanzarse de forma libre, autónoma y personalizada, de acuerdo con los propios intereses y proyectos cognoscitivos.

Los educadores, antes que continuar ejerciendo el rol de guardianes de la casa y de las tradiciones, hemos de asumir concientemente el rol de agentes de cambio. Ello implica no sólo la apertura hacia nuevas conceptualizaciones, sino la necesidad de ser garantes de la calidad que, entre otros mecanismos, es la mejor forma de evitar que la educación en general, pero sobre todo la educación virtual, se conviertan en una mercancía más, lo que desafortunadamente es hoy, fuerza es reconocerlo, también una tendencia en boga.

\section{LA CALIDAD DE LA EDUCACIÓN}

Aunque la preocupación por la calidad de la educación ha sido una constante histórica que se da individual, familiar e institucional, en nuestra región sólo se convierte en tema sistemático de estudio y análisis a partir de los ochenta, luego de los avances en los niveles de escolaridad de las dos décadas anteriores, cuando en la reunión de Ministros de América Latina y el Caribe de 1979, la declaran como tema prioritario.

Algo semejante sucede con la acreditación. Por lo menos en los términos explícitos y formales como se la conoce actualmente, es relativamente nueva en la cultura latinoamericana. De acuerdo con estudios nacionales y regionales recientes, es sólo desde los noventa cuando la evaluación de la calidad y la acreditación de instituciones y de programas académicos ha comenzado a ocupar un lugar central en las políticas públicas, se expiden normas específicas y se crean sistemas e instituciones encargados de adelantarlas, ante la necesidad de mejorar y/o transformar los sistemas educativos.

Históricamente, la acreditación o rendición social de cuentas ha sido consecuencia fundamental del desarrollo de una cultura y práctica común en las democracias participativas reales (frente a las meramente formales); de una cultura que desarrolla prácticas asociativas, como mecanismos para potenciar y hacer más eficaz la participación y la consecuente exigencia en el cumplimiento de deberes y derechos; de una cultura y prácticas del control efectivo de la calidad de los diferentes productos y servicios; de una cultura que aboca la necesidad de cambios reales, como forma para mejorar los desempeños y avanzar en el desarrollo individual y social.

Por las condiciones y tradiciones sociopolíticas en que se ha desenvuelto nuestra región latinoamericana, éstas no han sido precisamente características sobresalientes de nuestros países, y apenas comienzan a desarrollarse con relativa fuerza. En otras culturas, como la anglosajona, donde se cuenta con una mayor historia asociativa y participativa, la acreditación ha sido una práctica más antigua y arraigada.

El tema de la calidad de la educación en general y de la evaluación y acreditación como mecanismos para alcanzarla ha sido una de las preocupaciones centrales de la Unesco, tanto en educación básica como superior. Luego de haber declarado las décadas del sesenta y setenta como las de "la educación para el desarrollo", la década del ochenta se definió como la "década de la 
calidad de la educación". Diferentes estudios y conceptualizaciones dieron lugar al diseño de múltiples programas y mecanismos de evaluación ${ }^{14}$. Desde entonces, el tema ha ocupado lugar central en las diferentes cumbres y conferencias internacionales ${ }^{15}$, y se han venido impulsando diversas acciones.

En nuestra región, merece mencionarse el Laboratorio Latinoamericano de Evaluación de la Calidad de Educación para el caso de la educación básica y, en materia de educación superior, entre otros, los esfuerzos del Instituto Internacional para la Educación Superior en América Latina y el Caribe (IESALC) por incentivar la creación de una voluntad política en las instituciones para introducir en su gestión el rendimiento de cuentas, como un valor que sea reconocido por propios y extraños.

Los primeros resultados están a la vista. En educación básica, de una parte, se realizó hace unos años el Primer Estudio Internacional Comparativo y diferentes pruebas que, siguiendo el modelo educativo predominante que privilegia el desarrollo de un solo tipo de inteligencia (la lingüísticomatemática), se concentró en la realización de evaluaciones sobre "lenguaje, matemática y factores asociados". Y, en la actualidad, se prepara el segundo estudio de calidad, dentro de un enfoque más amplio y mul- tidimensional, que toma en consideración el cambio de paradigmas en marcha ${ }^{16}$. De otra parte, en educación superior, IESALC ha promovido la cultura de la evaluación y la acreditación; ha fomentado la realización de una serie de estudios nacionales sobre el tema, los cuales se encuentran disponibles y pueden ser consultados en línea en el Portal www.iesalc.unesco.org.ve; se cuenta con la Red de Evaluación y Acreditación en América Latina y el Caribe (RESALC), para compartir experiencias y acciones; y más recientemente, se ha formulado la propuesta de un "modelo de autoevaluación de programas"17 para facilitar la acreditación institucional y de programas de educación superior en los diferentes países de la región.

De acuerdo con el estudio regional de Orozco y Cardoso, los desarrollos alcanzados en materia de acreditación han sido desiguales, aunque se haya trabajado con esquemas metodológicos semejantes (autoevaluación, evaluación por pares y evaluación final por parte del organismo acreditador), pero diferentes en su forma de funcionamiento. En algunos países (como Argentina, Brasil y Venezuela) se ha avanzado más rápidamente que en otros, gracias a la obligatoriedad de la acreditación. En algunos, los análisis se focalizan en la acreditación de programas, en otros, en las instituciones. En unos casos las variables

14 Cfr. Facundo, A. y C. Rojas. La calidad de la educación secundaria. Lo que dice la investigación. Bogotá: Editorial MEN, 1982; y La calidad de la Educación: cómo entenderla y evaluarla. Bogotá: FES-Alegría de Enseñar, 1990.

15 Por ejemplo, la Conferencia Mundial de Educación de Calidad para Todos, de Jontiem o la Conferencia Mundial sobre la Educación Superior, realizada en París en 1998, para citar tan sólo las más recientes y significativas.

16 Cfr. Laboratorio Latinoamericano de Evaluación de la Calidad de la Educación - LLECE En: www. unesco.cl.

17 Cfr. Orozco, Luis Enrique y Rodrigo Cardoso. La evaluación como estrategia de autorregulación y cambio institucional. UNESCO-IESALC - RAP. Versión 0.0. Caracas: UNESCO-IESALC, febrero 2004. (Se puede consultar en línea, en la página del IESALC). 
y análisis utilizados son cuantitativos, en otros cualitativos. En ocasiones el acento se ha colocado en la búsqueda de estándares mínimos, en otras, en la identificación de estándares máximos. En muchos casos, se ha tenido más autoevaluación que acreditación, lo cual ha propiciado el control institucional interno. En otros, ha primado la acreditación, buscando que ésta incremente el control y la vigilancia gubernamentales. En algunos países se ha dado mayor presencia de los académicos tanto en los organismos como en sistemas de acreditación. Pero, en general, ha habido escasa presencia de los gremios profesionales en la definición de las políticas de acreditación.

La cultura de la evaluación y acreditación es aún más necesaria si se toma en consideración el fenómeno de la oferta globalizada de programas educativos y, más específicamente, las diferentes propuestas de integración bilateral y/o regional que se encuentran en plena realización y/o en marcha. Este fenómeno al tiempo que las hace necesarias, vuelve su conceptualización y práctica aún más complejas. Quizás pueda ser de aceptación universal afirmar que el objetivo fundamental de la acreditación es asegurar que la educación que proveen los programas y/o instituciones tenga niveles aceptables de calidad. Sin embargo, ello no está exento de dificultades teóricas y prácticas, particularmente en nuestra sociedad actual, en la cual no es fácil conciliar la tensión existente entre lo local y lo global. Cabe preguntarse, por ejemplo: ¿Frente a qué tipo de sociedad (local, global o una integración de ambas) deben ser aceptables los niveles de calidad?
De otra parte, son diversas las funciones o utilidades prácticas de la evaluación y acreditación de los programas y/o instituciones: la rendición de cuentas al Estado y a la sociedad, servir de mecanismo para la homologación de competencias, para los intercambios de estudiantes y docentes, para la cooperación entre programas e instituciones nacionales e internacionales, así como de forma de reconocimiento y publicidad para competir frente a otras instituciones. En cada una de esas funciones se abocan diversos tipos de problemas de compleja resolución. Me refiero solamente a dos, de diverso carácter: unos netamente académicos y otros extra académicos.

Desde el punto de vista académico puede decirse que el modelo básico de acreditación y de las evaluaciones descansa sobre conceptos como calidad, rendición de cuentas ante el Estado y la sociedad por considerarse la educación un servicio público, u otros como la eficiencia, efectividad o eficacia de la institución y/o del programa para cumplir con los objetivos que se propone, optimizando medios y recursos. Sin embargo, sucede que ninguno de éstos son conceptos unívocos ni tienen siempre el mismo referente. Por supuesto que pueden acordarse definiciones "consensuales". Por ejemplo, entender la calidad como el grado de congruencia o consistencia entre el contenido de la misión y la práctica diaria de la institución o programa académico que, de hecho, ha sido la definición más comúnmente empleada.

Sin embargo, de acuerdo con la Teoría del Control Total de Calidad, a este tipo de "calidad" se la conoce bajo el término de "calidad de concordancia" con el diseño previsto. Y, por supuesto, es apenas una
La cultura de la evaluación y acreditación es aún más necesariasi se toma en consideración el fenómeno de la oferta globalizada de programas educativos y, más específicamente, las diferentes propuestas de integración bilateral $y / o$ regional que se encuentran en plena realización $y / o$ en marcha. 
en otras dimensiones de calidad. Hay (o debe haber) igualmente una "calidad del diseño". Así las cosas, antes que suponer la calidad de un determinado diseño, habría que determinarla previamente. Incluso, de acuerdo con dicha teoría, se recomienda que precisamente cuando hay deficiencias generalizadas u otras razones, se aconseja determinar la calidad de diseño antes que la calidad de consistencia. Y, muchas veces, el sólo cambio de diseño o de "modelo" frente al cual hacen las demás evaluaciones, es suficiente para corregir las deficiencias y mejorar el estado de cosas.

Si se analiza con detenimiento la situación actual de la educación, pareciera que precisamente tales características se estuvieran dando. Las deficiencias y tensiones no parecen ser sólo propias de la congruencia misión-práctica educativa, sino que se tienen en la misma determinación de la misión educativa, frente a la cual se hacen las evaluaciones de consistencia. Por ejemplo, las tensiones entre lo local y lo global, o entre los enfoques del aprendizaje heterónomo y regulado, frente al autónomo y autorregulado parecen ser tanto o más carencias del "modelo" de educación por seguir que de la sola práctica educativa diaria. Algo semejante sucede con la selección de las características por evaluar.

Igualmente ha existido y continúa el debate en torno a si los criterios que se van a utilizar deben ser diferenciales para programas presenciales y a distancia. Debate que yo considero debería referirse más bien, como se expuso ampliamente arriba, a los diferentes paradigmas y modelos educativos existentes en la actualidad: heterónomos o autónomos, tradicionales o con integración o manejo de espacios virtuales. Es apenas obvio que de los paradigmas que se tengan como referentes dependen los resultados de las evaluaciones.

El segundo caso que traigo a colación, hace referencia a posibles debilidades no académicas, pero reales y prácticas, y puede servir para reflexionar sobre las funciones de las entidades o agencias acreditadoras, así como sobre las reglas y ámbitos de competencia (nacional, regional o transnacional) que se les atribuyan.

De una parte, una de las debilidades de las prácticas de la acreditación anotadas por el estudio de Orozco y Cardoso es precisamente la escasa presencia de asociaciones educativas en las instituciones acreditadoras que, en la práctica, merma reconocimiento a las acreditaciones, ya que quienes expiden los criterios y conducen las evaluaciones de los "pares" no tendrían el suficiente peso y representación de la comunidad académica, bien sea nacional o internacional. Esa es una gran diferencia con el caso de países, como los Estados Unidos, donde las agencias acreditadoras son comunidades académicas de amplia representación, y cuya autoridad y calidad debe ser probada y reconocida previamente por la Secretaría de Educación, por períodos no superiores a cuatro años. Cuando tales condiciones no se dan, quien termina expidiendo los criterios, conduciendo las evaluaciones de pares y, finalmente, respaldando la acreditación no es el sector académico, sino un pequeño comité o incluso, el mismo Estado.

De otra parte, y como forma indirecta de ilustración, permítanme mencionarles un caso no propiamente del área educativa, que ha concitado un gran debate entre jueces y 
economistas, con motivo de las prácticas y análisis de la integración regional.

Los antecedentes más importantes que en esta materia se tienen en la región, son el acuerdo trilateral NAFTA y el Acuerdo de Libre Comercio con Chile. Allí, existen reglas específicas como las denominadas de "protección de inversiones", que fueron concebidas como un mecanismo útil para "blindar" las inversiones transnacionales contra posibles nacionalizaciones o confiscaciones. Y, si bien son fundamentalmente aplicables a otros campos, por supuesto también lo pueden ser en el educativo.

De acuerdo con algunos autores, los casos que hasta el presente han sido sometidos al mecanismo denominado de "inversionista contra Estado" durante la vigencia del NAFTA, nada han tenido que ver con las posibles confiscaciones que dieron origen a las reglas. Por el contrario, las disputas se han relacionado con la expedición de leyes, regulaciones y medidas concretas tomadas por los gobiernos en el ámbito nacional, estatal o local que, según los demandantes, violan el acuerdo en materia de inversiones. En virtud de tal mecanismo, se ha producido un giro en los ejes del arbitramento internacional hasta ahora conocido, que sólo ventilaban casos de transacciones comerciales. Bajo el NAFTA, los denominados "tribunales de protección de inversiones" se han ocupado - en la práctica- de casos relacionados con la conducción de las políticas públicas en los países miembros, sometidos así al examen y dictamen de un sistema transnacional de arbitraje que puede castigar fuertemente las finanzas estatales, y desafía -hasta cierto punto- las tradicionales autonomías políticas (incluso judiciales) nacionales, además sus deliberaciones son a puerta cerrada. Chile logró algunos cambios que favorecen el conocimiento público de las demandas en curso, pero el sistema mencionado sigue en pie.

Pues bien, tales demandas: "inversionista contra Estado" han producido diversas tensiones. En los Estados Unidos se ha llegado a comentar, incluso, que dichos mecanismos son la mayor amenaza sobre la independencia judicial de la nación, de la cual se tenga noticia, ya que el organismo que toma la decisión es un tribunal especial de arbitramento, elegido por las partes en conflicto, y está facultado para conceder indemnizaciones con cargo al erario del país que resulte condenada a pagarlas. Estos tribunales están facultados para revisar, desconocer o rechazar fallos judiciales o la legislación nacional. Como decía un comentarista, no se trata de un asunto de poca monta.

Estos dos casos son solamente muestra de que las rupturas y cambios de paradigmas tienen diversas repercusiones, ofrecen nuevas posibilidades, pero igualmente presentan problemas y dificultades en las más diferentes áreas que, al contrario de llevarnos al inmovilismo, han de movernos a la búsqueda de nuevas hipótesis y alternativas de solución.

\section{LA ACREDITACIÓN EN LA EDUCACIÓN A DISTANCIA Y VIRTUAL}

Aunque la educación a distancia es casi tan antigua como nuestra civilización occidental, la aparición de la educación a distancia en la región no sólo fue tardía, sino que su evolución histórica, tecnológica y conceptual ha sido lenta, difícil y asimétrica. Y, consecuentemente, ello se ha reflejado
Aunque la educación a distancia es casi tan antigua como nuestra civilización occidental, la aparición de la educación a distancia en la región no sólo fue tardía, sino que su evolución histórica, tecnológica y conceptual ha sido lenta, dificily asimétrica. 
también en la acreditación de programas e instituciones.

Así, por ejemplo, mientras que en Europa y Estados Unidos la educación a distancia inició sus desarrollos formales desde la segunda mitad del siglo XIX y ya desde 1938 se había conformado el Consejo Internacional para la Educación por Correspondencia (International Council for Correspondence Education) que en la actualidad se conoce como el International Council for Open and Distance Education (ICDE), en nuestra región latinoamericana los primeros desarrollos de la educación superior a distancia se iniciaron originalmente en México, apenas en 1947, con algunas actividades de capacitación del Instituto Federal de Capacitación del Magisterio.

De otra parte, se hace necesario reconocer que durante mucho tiempo esta modalidad se asoció con un tipo de educación de baja calidad, debido a que los primeros desarrollos relativamente masivos de la educación por correspondencia se dieron en los programas de capacitación de adultos como el físicoculturismo (los conocidos cursos por correspondencia de Charles Atlas), la mecánica popular, electrónica, radio y televisión, periodismo, dibujo publicitario por correspondencia, etc. que, por tener un carácter "trivial, comercial, banal y puramente lucrativo", generaron prejuicios sociales y no pocas resistencias.

Así las cosas, cuando por iniciativa de gobiernos u otras entidades y bajo el patrocinio del Instituto Latinoamericano de
Comunicación Educativa de la Unesco, esta modalidad llega inicialmente a la educación básica (donde se han realizado diversas experiencias masivas muy exitosas ${ }^{18}$ ) $y$, posteriormente, a la educación superior, se debieron enfrentar dichos prejuicios y resistencias, lo que -sin lugar a dudashicieron más tardío y difícil el desarrollo de esta importante modalidad educativa en el nivel superior. Prácticamente sólo en los años setenta y ochenta se crean en los diversos países programas de educación superior a distancia en instituciones convencionales e incluso algunas pocas instituciones especializadas, siguiendo diversas experiencias de países desarrollados ${ }^{19}$.

Los prejuicios mencionados así como la escasa incorporación e integración de los nuevos programas con los cuerpos docentes constituidos y la utilización de lo que ahora puede denominarse como "bajas tecnologías" de entrega de contenidos (fundamentalmente materiales impresos y muy posteriormente con alguna combinación multimedia incluso digitalizada) y sobre todo la utilización de enfoques lineales de instrucción programada de exposiciónrecepción, han sido verdaderos lastres en materia para la acreditación social de esta modalidad. Con todo, diversos programas e instituciones de educación a distancia de diversos países alcanzaron en tiempo relativamente corto no sólo reconocimiento nacional, sino regional e internacional.

Una nueva etapa de desarrollo se

18 Por ejemplo, la telesecundaria y el bachillerato de Estado de México, las escuelas radiofónicas y la televisión educativa de Colombia o la Radio Escola y TV Escola de Brasil, así como otras experiencias en países de Centro América.

19 En la región tienen gran impacto las experiencias de la Open University de Inglaterra, de la UNED de España, de la Radio Nederland de Holanda y en menor grado de la Fern Universität de Alemania. 
inició desde finales de los ochenta. En 1989, el Instituto Tecnológico de Estudios Superiores de Monterrey - ITESM realizó las primeras pruebas de internet en la región y, a partir de entonces, comenzó la rápida expansión de esta tecnología en nuestros países. Con ella, y con la amplia comercialización de los computadores personales y la multimedia, se introdujo en la región lo que se denomina la educación a distancia electrónica (Electronic Distance Educación). Con las tecnologías digitales y los reconocimientos logrados por la educación a distancia, los anteriores prejuicios comenzaron a ceder rápidamente, y desde entonces no solo se han venido utilizando las tecnologías digitales como apoyo de los programas convencionales, sino que han servido de plataforma para explorar el ofrecimiento de programas de educación virtual $^{20}$.

Un primer análisis realizado sobre la educación virtual en la región, que tuve el honor de realizar por encargo de IESALC, mostró que el desarrollo inicial de esta modalidad se concentró fundamentalmente en el ofrecimiento de programas de educación continuada (como en caso de Colombia) o de postgrados (como en el caso de México y Chile), o incluso de programas de pregrado (como en caso de Argentina, y posteriormente de la mayoría de los países). Por supuesto que estos desarrollos no han sido generalizados y son desiguales entre y dentro de los países ${ }^{21}$.

Los esfuerzos por acreditar la educación superior, han estado igualmente pre- sentes en estas modalidades alternativas. Así por ejemplo, IESALC ha promovido diversos seminarios nacionales e internacionales e igualmente lo han hecho asociaciones especializadas como la Asociación Iberoamericana de Educación Abierta y a Distancia AIESAD, el ya mencionado ICDE (que cuenta con un capítulo regional muy activo) y los seminarios internacionales que organiza INNOVA de la Universidad de Guadalajara. En cada una de estas organizaciones, para mencionar tan sólo unas pocas, la preocupación por la calidad, la evaluación y la acreditación se ha venido planteando de forma sistemática como una de las prioridades de instituciones y países. Incluso desde julio del 2002, se constituyó la Red Iberoamericana para la Evaluación y la Acreditación de la Calidad de la Educación Superior- RIACES. Estas, y las diversas acciones específicas que adelantan las agencias acreditadotas tienen un impacto positivo sobre el mejoramiento de la calidad de la educación a distancia y virtual.

De otro lado, si se considera que, no obstante las dificultades y diferentes niveles de desarrollo de las instituciones que ofrecen educación a distancias, son precisamente los programas e instituciones de educación a distancia quienes han realizado quizás los mayores avances en el proceso de rupturas y cambios de paradigmas antes mencionados, la situación es aún más promisoria. En efecto. Si bien es cierto que en la educación a distancia e incluso virtual predominan aún los diseños ins-

20 Facundo, A. La Educación Superior Abierta y a Distancia: Necesidades para su Establecimiento y Desarrollo en America Latina y el Caribe. Informe para UNESCO-IESALC. Bogotá, febrero 2002.

21 Facundo, A. Educación Virtual en América Latina y El Caribe: Características y Tendencias. Informe para UNESCO-IESALC. Bogotá, febrero 2002. 
La virtualidad más que la simple incorporación de las tecnologías digitales, implica la adopción de nuevos métodos pedagógicos, nuevas actitudes $y$ hábitos ante el aprendizaje. La verdadera universidad virtual todavía no existe aún en ninguna parte del mundo. truccionales lineales y buena parte de los esfuerzos se han centrado en la producción y entrega de materiales (escritos, audiovisuales, multisensoriales o digitales) y en la reproducción en estos nuevos medios del modelo de presentación programada y regulada de contenidos siguiendo el viejo paradigma de aprendizaje por exposición y recepción, también lo es que ha sido precisamente en los programas de educación a distancia, y en especial en aquellos que utilizan plataformas virtuales, donde más se ha venido ganando en flexibilidad, apertura de nuevos espacios de aprendizaje e, incluso en la exploración y gradual incorporación de las grandes posibilidades del autoaprendizaje; en el cambio de funciones de estudiantes y docentes, la mayoría de los cuales se concentran en el apoyo, tutoría y exámenes continuos a sus estudiantes; en el fomento del aprendizaje colaborativo, así como en la autoevaluación de los aprendizajes, y no tanto en la entrega gradual de contenidos.

Además, y tal como lo indican diversos autores internacionales, son las modalidades de educación a distancia y la denominada educación virtual quienes están más cerca de alcanzar la meta pedagógica no sólo de aprendizajes autónomos sino autodirigidos. Por primera vez en la historia del aprendizaje -afirma Peters- en los ambientes digitalizados "el aprendizaje autónomo podrá ser desarrollado, aplicado y practicado con relativa facilidad. Podrá ser realizado el viejo ideal pedagógico del estudiante independiente, autogestor, autocompetente, con confianza en sí mismo, que aprende por su cuenta"22.
Con todo, todavía es largo el camino por recorrer, hasta lograr una verdadera ruptura en los paradigmas, incluso en la denominada "educación virtual”. De acuerdo con expertos, en la actualidad se está abusando del término "virtual", bien sea para presumir o engañar. Existen cursos, seminarios o programas que usan los computadores, la multimedia y el internet, pero sólo para mejorar las presentaciones, para distribuir más velozmente los materiales o para "impartir" conocimientos mejor empaquetados. No han cambiado la estructura básica de enseñanza por exposición, continúan apegados a las estructuras pedagógicas convencionales y no utilizan todo el potencial que brindan las tecnologías digitales para buscar información rompiendo las restricciones de distancia, tiempo y hasta de la propia realidad; para aprender investigando, para visualizar desde las más diversas dimensiones; para utilizar la comunicación y la colaboración como forma de enriquecer los procesos de aprendizaje; o para que el estudiante asuma la responsabilidad por un proceso de aprendizaje autónomo, consciente, permanente y dirigido en lo fundamental por él mismo.

La virtualidad más que la simple incorporación de las tecnologías digitales, implica la adopción de nuevos métodos pedagógicos, nuevas actitudes y hábitos ante el aprendizaje. La verdadera universidad virtual todavía no existe aún en ninguna parte del mundo. Parece abrirse el consenso de que, además de realizar todas las funciones que se atribuyen a la educación superior (investigación, docencia y proyección social), una universidad virtual ha 
de entenderse, como lo sostienen Aiko y Pogroszewski, como una "infraestructura que brinde a los estudiantes una experiencia de aprendizaje y servicios de apoyo para completar una carrera y a los miembros del cuerpo docente los recursos para enseñar e investigar eficazmente en línea"23.

Todos y no solamente algunos de estos nuevos elementos han de ser tomados en consideración para evaluar la calidad de los programas. Y como decía anteriormente, no basta con que los pares y los organismos de acreditación garanticen la "calidad de conformidad". Ello, sin duda alguna, es un avance. Empero, debemos preocuparnos igual o quizás prioritariamente por la "calidad de los diseños", de los enfoques que se reflejan en la misión, en los planes educativos institucionales y, por supuesto, en la práctica educativa diaria.

Todos sabemos que se trata de un reto difícil y complejo. Sin embargo, si se toma conciencia sobre el particular, los esfuerzos que se vienen realizando cobrarán una nueva dimensión y lograrán los avances significativos que todos anhelamos. Sólo entonces podrán contribuir las plataformas virtuales a transformar realmente la educación, a producir la revolución copernicana que se prevé.
La gran tarea que tiene la evaluación y acreditación de la educación a distancia y virtual es contribuir a que los programas hagan un salto hacia niveles superiores de calidad en los aprendizajes, los cuales, en concepto de los expertos, requieren y significan una verdadera ruptura y cambio de paradigmas.

Como nunca antes en la historia, en la actualidad además contamos ya con las plataformas virtuales, con los conocimientos pedagógicos y con mecanismos institucionales -como la evaluación y acreditación de la calidad- que hacen posible alcanzar la vieja aspiración de motivar a los estudiantes a asumir de forma consciente y con los medios digitales actualmente disponibles, la responsabilidad de su aprendizaje no sólo durante un período de su vida, sino como una cultura y un hábito que se desarrolla de forma continua, a lo largo de toda la vida.

El camino natural del aprendizaje: aprender investigando. Es en esa dirección como debemos movernos los docentes, los programas y las instituciones. Si lo logramos, habremos producido una verdadera revolución educativa. Esa sería una verdadera agenda de "modernización" de la educación. No me cabe duda que ustedes avanzarán en esa dirección.

23 Cfr. Auki, K. y D. Pogroszewski. "Virtual University Referente Model: A Guide to Delivering Education and Support Services to the Distance Learner". En: http://www.westga.edu/ distance/aoki13.html. 